"tmcs-gofen" — 2013/10/21 — 15:20 — page 241 — \#1

\title{
Powers which commute or associate as solutions of ODEs
}

\author{
Alexander Gofen
}

\begin{abstract}
This paper is dedicated to the two classical transcendental functions: The locus of points for which powers commute, and the locus of points for which powers associate. These classical functions however are considered in a new perspective: as holomorphic solutions of ODEs passing over the points of singularity of these ODEs.

Generally, solution functions which are holomorphic at singular points of the phase space of ODEs were studied in [2,3], and it was shown in [3], that certain holomorphic functions may satisfy only singular rational ODEs. This is the frame in which the function of commuting or associating powers are considered in this paper.

First we obtain several types of ODEs satisfied by these functions. The obtained ODEs happen to have singular points, yet the solutions are proved to be holomorphic at these points, and their Taylor expansions are obtained. However it is not yet known whether these two transcendental functions can satisfy a regular rational ODE at the respective special points. The article also poses an open question about remarkable inequalities related to the commuting powers.
\end{abstract}

Key words and phrases: commuting powers, associating powers, singular ODE, regular singularity.

ZDM Subject Classification: I20, I70, R20.

\section{Introduction}

Operation of raising into power generally is neither commutative nor associative. However for certain subsets of real numbers the powers do commute or associate, and the respective locuses are defined via transcendental functions having remarkable graphs (see below). These classical functions have been known and studied for a long time, as it is summarized in the review [1].

Copyright (c) 2013 by University of Debrecen 


$$
\text { "tmcs-gofen" — 2013/10/21 — 15:20 — page } 242 \text { — \#2 }
$$

Here we are going to study these functions from the view point presented in [2], Section 8, 8.2: To obtain ODEs satisfied by these functions, and to evaluate their Taylor coefficients at singular points of the phase space.

Originally, both functions are defined by implicit transcendental equations, but they also satisfy certain elementary ODEs [2]. For both of them the ODEs have a point of singularity at which the solutions however are regular. These are the so called regular singularities already introduced and studied in our previous works [2].

Along this paper, the concepts of differentiability and holomorphy are understood as in the complex space.

Some important ODEs and examples considered in this paper may be displayed, and experiments with them may be made using the free software referred in Appendix 1.

\section{Commuting powers}

We are considering pairs $(x, y)$ of real numbers $x, y>0$ for which

$$
x^{y}=y^{x} \text {, or } F(x, y)=x^{y}-y^{x}=0 .
$$

The trivial solution of this transcendental equation is identity function $y=x$ indeed. Yet this equation must have also a non-trivial solution: for example the points $(2,4)$ and $(4,2)$ satisfy $(2.1)$ and at those points the partial derivatives

$$
\frac{\partial F}{\partial y}=x^{y} \ln x-x y^{x-1}=x^{y} \ln x-y^{x} \frac{x}{y}=x^{y}\left(\ln x-\frac{x}{y}\right)
$$

exist and are nonzero, so that the nontrivial solution $y(x)$ surely exist in a neighborhood of those points (see Figure 1).

Observe that at one particular point $(e, e)$ the partial derivative $\left.\frac{\partial F}{\partial y}\right|_{(e, e)}=0$ meaning that $(e, e)$ is a singular point of the equation (2.1) - subject of a special study in this article.

Further on, $y(x)$ denotes only the non-trivial solution of equation (2.1). As it was shown in [1], equation (2.1) admits a remarkable parametric solution

$$
x=\left(1+\frac{1}{t}\right)^{t}, \quad y=\left(1+\frac{1}{t}\right)^{t+1}
$$

which in particular delivers infinitely many rational solutions of (2.1) and the above mentioned $(2,4)$ and $(4,2)$ - the only integer solutions. 
Not touching the various properties of the equation (2.1) well covered in [1], here we are looking for representations of the $y(x)$ as a solution of certain ODEs and then obtaining its (formal) expansion into a Taylor series at the special point $(e, e)$.

THEOREM 1. Function $y(x)$ satisfies the ODEs

$$
y^{\prime}=\frac{\frac{y}{x}-\ln y}{\frac{x}{y}-\ln x}=\left(\frac{y}{x}\right)^{2} \frac{\ln \frac{x}{e}}{\ln \frac{y}{e}}
$$

regular at all points of the phase space except the point $x=y=e$.

Proof. Differentiate the equation (2.1)

$$
0=y^{\prime} x^{y} \ln x+y x^{y-1}-y^{x} \ln y-y^{\prime} x y^{x-1}=y^{\prime} x^{y} \ln x+\frac{y}{x} x^{y}-y^{x} \ln y-y^{\prime} \frac{x}{y} y^{x} .
$$

Dividing by $x^{y}=y^{x} \neq 0$ we get

$$
0=y^{\prime} \ln x+\frac{y}{x}-\ln y-y^{\prime} \frac{x}{y}
$$

yielding both required formulas (2.3). If $y \equiv x$, the ODE reduces to a trivial one $y^{\prime} \equiv 1$. For a non-trivial $y(x)$, this $\mathrm{ODE}$ is singular at the point $(e, e)$.

Therefore both trivial and non-trivial solutions are holomorphic at all points except possibly $(e, e)$. The identical solution is holomorphic also at this point indeed. As to $y(x)$, we will establish that it is holomorphic at this point later.

The ODE (2.3) is not polynomial. In the next Theorem we will obtained a polynomial ODE satisfied by $y(x)$.

THEOREM 2. The function $y(x)$ satisfies the polynomial ODE

$$
y^{\prime \prime} x^{2} y^{2}(y-x)-\left(y^{\prime}\right)^{3} x^{4}+\left(y^{\prime}\right)^{2} y x^{2}(3 x-2 y)+y^{\prime} y^{2} x(3 y-2 x)-y^{4}=0 .
$$

Proof. From $y^{x}=x^{y}$ we get $y=x^{\frac{y}{x}}$. Differentiating it we obtain

$$
y^{\prime}=\frac{y}{x} x^{\frac{y}{x}-1}+\frac{y^{\prime} x-y}{x^{2}} x^{\frac{y}{x}} \ln x=y\left(\frac{y}{x^{2}}+\frac{y^{\prime} x-y}{x^{2}} \ln x\right) .
$$

Solving the latter equation for $\ln x$ we get that

$$
\ln x=\frac{y^{\prime} x^{2}-y^{2}}{y^{\prime} y x-y^{2}} .
$$

Differentiating again and rearranging the equation we get the ODE (2.4). 


$$
\text { "tmcs-gofen" — 2013/10/21 — 15:20 — page } 244 \text { — \#4 }
$$

REMARK 1. ODE (2.4) is also singular at the point where $y=x$. At this point the monomial with $y^{\prime \prime}$ disappears, while for the value $\left.y^{\prime}\right|_{x=e}=a$ we get from (2.4) that

$$
\left(-a^{3}+a^{2}+a-1\right) e^{4}=0 \quad \text { or }\left(a^{2}-1\right)(a-1)=0
$$

yielding two possible values for $\left.y^{\prime}\right|_{x=e}= \pm 1$. For the nontrivial solution $\left.y^{\prime}\right|_{x=e}=-1$.

\subsection{Evaluation of the formal Taylor expansion at the point $(e, e)$}

At every regular point $(x, y)$ of $(2.3)$ the solution $y(x)$ is holomorphic, and all its derivatives may be evaluated via differentiation of equation (2.1) or (2.3). Evaluation of the Taylor coefficients of the solution may be possible also at singular points of the phase space of ODEs by analyzing and solving the so called generating algebraic system (in which the polynomial ODE (2.4) would be the first equation). As it follows from the Remark, the equation (2.4) has exactly two solutions - the components of two possible Taylor expansions: $(e, 1,0,0,0, \ldots)$ corresponding to the trivial solution, and $\left(e,-1, a_{2}, a_{3}, \ldots\right)$ corresponding to the nontrivial solution. However the polynomial ODE (2.4) is too complicated for evaluation of the components $a_{2}, a_{3}, \ldots$.

To avoid cumbersome transformations and computations, it makes more sense to differentiate (2.1) by applying the formulas of automatic differentiation (AD) [4], [5] to the simplest of the available equation for $y(x)$ :

$$
x \ln y=y \ln x \quad \text { or } \quad x v=y \ln x, \quad \text { where } v=\ln y .
$$

The AD formulas operate with the so called normalized derivatives denoted with square brackets: $y^{[n]} \stackrel{\text { def }}{=} \frac{y^{(n)}}{n !}$. (Normalized derivatives are in fact the respective Taylor coefficients). According to the AD formulas,

$$
\begin{array}{rlrl}
v^{[n]} & =\frac{1}{y}\left(y^{[n]}-\sum_{i=1}^{n-1}\left(1-\frac{i}{n}\right) y^{[i]} v^{[n-i]}\right), & & n \geq 1 \\
(x v)^{[n]} & =x v^{[n]}+v^{[n-1]}, & n \geq 2 \\
(y \ln x)^{[n]} & =y^{[n]} \ln x+\sum_{i=1}^{n} \frac{y^{[n-i]}(-1)^{i-1}}{i x^{i}}, & & n \geq 1 .
\end{array}
$$

Continuing the process of differentiation at the point $x=e$ applying these formulas, we see that beginning from $n \geq 2$ the highest available derivative $y^{[n+1]}$ 
appears only in a linear algebraic equations and may be readily obtained.

$$
\begin{gathered}
y^{[n+1]}=\sum_{i=0}^{n} \frac{y^{[n-i]}}{(i+2)(-e)^{i+1}}+ \\
+\frac{2 m+3}{n+2} \sum_{i=1}^{n}\left(1-\frac{i}{n+1}\right) y^{[i]} v^{[n+1-i]}+e \sum_{i=2}^{n}\left(1-\frac{i}{n+2}\right) y^{[i]} v^{[n+2-i]}, \quad n \geq 1 . \\
v^{[n+1]}=\frac{1}{y}\left(y^{[n+1]}-\sum_{i=1}^{n}\left(1-\frac{i}{n+1}\right) y^{[i]} v^{[n+1-i]}\right)
\end{gathered}
$$

The first 15 values of $\left.y^{[n]}\right|_{x=e}$ and their structure are shown in the Table below.

\begin{tabular}{|c|c|c|c|}
\hline$n$ & $\left.y^{[n]}\right|_{x=e}$ & Rational factors only & Values \\
\hline 1 & -1 & & -1 \\
\hline 2 & $\frac{5}{3} \frac{1}{e}$ & $\frac{5}{3}$ & 0.6131324019524038693258730 \\
\hline 3 & $-\frac{25}{9} \frac{1}{e^{2}}$ & $\frac{5^{2}}{3^{2}}$ & -0.3759313423239241441499986 \\
\hline 4 & $\frac{1243}{270} \frac{1}{e^{3}}$ & $\frac{11 \cdot 113}{2 \cdot 3^{3} \cdot 5}$ & 0.2292049110416847449011949 \\
\hline 5 & $-\frac{1229}{162} \frac{1}{e^{4}}$ & $\frac{1229}{2 \cdot 3^{4}}$ & -0.1389501246558907875369102 \\
\hline 6 & $\frac{14107}{1134} \frac{1}{e^{5}}$ & $\frac{14107}{2 \cdot 3^{4} \cdot 7}$ & 0.08382029833871136184501299 \\
\hline 7 & $-\frac{575927}{28350} \frac{1}{e^{6}}$ & $\frac{11 \cdot 41 \cdot 1277}{2 \cdot 3^{4} \cdot 5^{2} \cdot 7}$ & -0.05035556630867463165817052 \\
\hline 8 & $\frac{4217764}{127575} \frac{1}{e^{7}}$ & $\frac{2^{2} \cdot 1054441}{3^{6} \cdot 5^{2} \cdot 7}$ & 0.03014777916178779933005792 \\
\hline 9 & $-\frac{1408003}{26244} \frac{1}{e^{8}}$ & $\frac{211 \cdot 6673}{2^{2} \cdot 3^{8}}$ & -0.01799772848935453347797715 \\
\hline 10 & $\frac{18804662561}{216513000} \frac{1}{e^{9}}$ & $\frac{149 \cdot 1091 \cdot 115679}{2^{3} \cdot 3^{9} \cdot 5^{3} \cdot 11}$ & 0.01071843133007776772407456 \\
\hline 11 & $-\frac{4465808232533}{31827411000} \frac{1}{e^{10}}$ & $\frac{41 \cdot 10949 \cdot 9948137}{2^{3} \cdot 3^{10} \cdot 5^{3} \cdot 7^{2} \cdot 11}$ & -0.006370212773188646017307121 \\
\hline 12 & $\frac{561757387253483}{2482538058000} \frac{1}{e^{11}}$ & $\frac{281 \cdot 1999136609443}{2^{4} \cdot 3^{11} \cdot 5^{3} \cdot 7^{2} \cdot 11 \cdot 13}$ & 0.003779319220659389337334455 \\
\hline 13 & $-\frac{553820639666903}{151992126000} \frac{1}{e^{12}}$ & $\frac{3719 \cdot 14891654737}{2^{4} \cdot 3^{12} \cdot 5^{3} \cdot 11 \cdot 13}$ & -0.002238794670049279589672062 \\
\hline 14 & $\frac{6546034449396991}{11171421261000} \frac{1}{e^{13}}$ & $\frac{6546034449396991}{2^{3} \cdot 3^{13} \cdot 5^{3} \cdot 7^{2} \cdot 11 \cdot 13}$ & 0.001324468375097206276459089 \\
\hline 15 & $-\frac{52573598131492979}{55857106305000} \frac{1}{e^{14}}$ & $\frac{8855401 \cdot 5936896379}{2^{3} \cdot 3^{13} \cdot 5^{4} \cdot 7^{2} \cdot 11 \cdot 13}$ & -0.0007826480747899546369361984 \\
\hline
\end{tabular}

Table 1. The Table ${ }^{1}$ of the Taylor coefficients of $y(x)$ at point $(e, e)$ obtained via the formulas $(2.1)$

The formulas (2.1) are recursive finite difference equations for obtaining the components of the solution-vector (and we do not know if the finite convoluted formula exists). Therefore, having only the recursive formula of the coefficients, we cannot determine whether the convergence radius of this Taylor expansion is nonzero (nor can we even make a guess about the general term observing these available 15 factorized coefficients).

${ }^{1}$ Courtesy to Lòczi Lajos who obtained the multi-digit rational coefficients of the expansion and their factorization using the symbol-processing software "Maple". 
We will establish that $y(x)$ is in fact holomorphic at $x=e$ in the following Theorem.

ThEOREM 3. Function $y(x)$ is holomorphic at the point $x=e$.

Proof. Satisfying the ODE (2.3), functions $y(x)$ is differentiable and holomorphic in a neighborhood of the point $(e, e)$ except may be this point itself. In order to establish that $y(x)$ is holomorphic also at this point, according to the Riemann Removable Singularities Theorem ${ }^{2}$ it is enough to prove that $y(x)$ can be continuously extended over the point $(e, e)$. Recalling the parametric representation (2.2), both limits below do exist

$$
\lim _{t \rightarrow \infty} y(t)=\lim _{t \rightarrow \infty}\left(1+\frac{1}{t}\right)^{t+1}=e ; \quad \text { and } \quad \lim _{t \rightarrow \infty} x(t)=\lim _{t \rightarrow \infty}\left(1+\frac{1}{t}\right)^{t}=e
$$

and do approach the point $(e, e)$, which proves the Theorem.

Therefore we have established that function $y(x)$ is holomorphic at $x=e$ without direct analysis of the general term of its Taylor expansion (which is unavailable). For this reason alone we cannot apply the method presented in [3] for trying to prove that $y(x)$ can satisfy no regular rational or polynomial ODE at this point. This question remains open.

Now take a look at the graph of $y(x)$ (Figure 1$)$ :

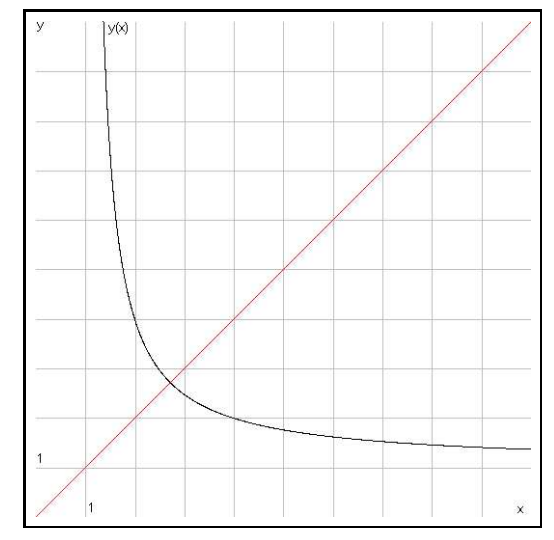

Figure 1. The trivial (red) and non-trivial (black) solution of $x^{y}=y^{x}$

$2_{\text {http://www .proofwiki.org/wiki/Riemann_Removable_Singularities_Theorem }}$ 
For points $(a, b)$ of the plane above the curve $y(x), a^{b} \geq b^{a}$ equality being reached if $a=b: 32=2^{5}>5^{2}=25$.

For points $(a, b)$ of the plane below the curve $y(x), a^{b} \leq b^{a}: 8=2^{3}<3^{2}=9$.

It is easy to observe that the function $y(x)$ resembles hyperbola (yet not coincides with it indeed). The unique hyperbola passing through the point $(e, e)$ and having the same asymptotes $y=1$ and $x=1$ does exist

$$
h(x)=\frac{(e-1)^{2}}{x-1}+1, \quad h^{\prime}=-\left(\frac{h-1}{e-1}\right)^{2}=-\left(\frac{e-1}{x-1}\right)^{2}
$$

and its graph looks remarkably close to $y(x)$ (Figure 2):

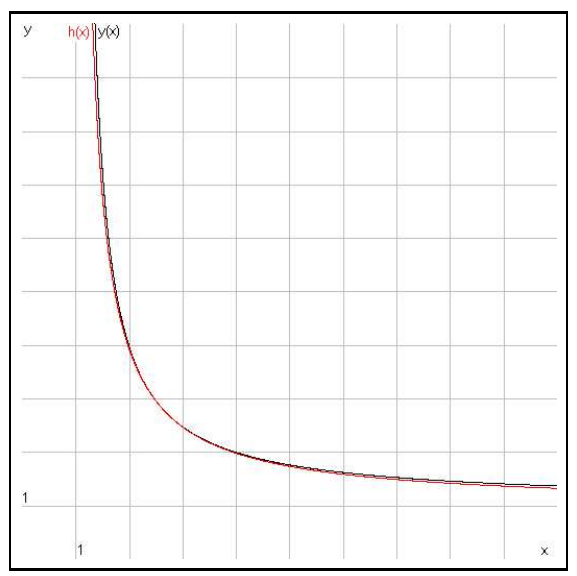

Figure 2. The hyperbola (red) passing through the point $(e, e)$ and having the same asymptotes $y=1$ and $x=1$ as the function $y(x)-$ the solution of $x^{y}=y^{x}$ (black)

\subsection{The remarkable inequalities}

Observe that the defining equation $x^{y}=y^{x}$ may be transformed into the form $x^{1 / x}=y^{1 / y}$, therefore let us take a closer look at the function $\mu(x)=x^{1 / x}$.

Consider the graph of $\mu(x)$ on the segment $[1, \infty)$. This function reaches its maximum at the point $\left(e, e^{1 / e}\right) \simeq(2.718,1.444)$ - because $\mu^{\prime}(x)=x^{\frac{1}{x}-2}(1-\ln x)$ changes its sign at the point $x=e$ so that $\mu(x)$ increases on $[1, e]$ and decreases on $[e, \infty)$. At that $\mu(1)=1$ and $\lim _{x \rightarrow \infty} \mu(x)=1$.

Function $\mu(x)$ therefore is comprised of two monotonic pieces: the increasing $\mu_{1}:[1, e] \rightarrow\left[1, e^{1 / e}\right]$ and the decreasing $\mu_{2}:[e, \infty) \rightarrow\left[1, e^{1 / e}\right]$. Then $\mu_{1}^{-1} \circ \mu_{1}$ and 
$\mu_{2}^{-1} \circ \mu_{2}$ stand for the trivial solution, while $\mu_{2}^{-1} \circ \mu_{1}$ and $\mu_{1}^{-1} \circ \mu_{2}$ stand for the nontrivial $y(x)$.

Geometrically, the function $\mu(x)$ generates commuting powers $x^{y}=y^{x}$ in the following way. Consider any abscissa $B$ to the right of $e$ so that $O B=b>e$ (Figure 3) with the corresponding ordinate $B C$. To the left of $e$ there may be one and only one equal ordinate $A E$ of the function $\mu(x)$. The abscissas $O A$ and $O B$ therefore comprise a solution pair $(x, y)$ for equation (2.1) of commuting powers: $O A=x, O B=y$ or vice versa.

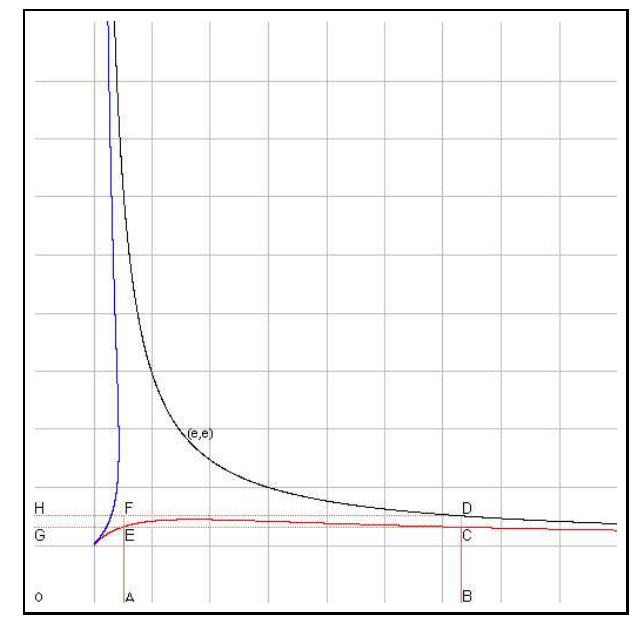

Figure 3. Function $y(x)$ (black) outlined by $\mu(x)=x^{1 / x}$ (red) and $\nu(y)=y^{1 / y}$ (blue)

Lemma 1. $y(x)>x^{1 / x}$.

Proof. The point $D$ on the curve $x^{y}=y^{x}$ corresponds to the abscissa $O B$ and ordinate $O H=O A$. At that $B C=A E=O G<O H$ because $A E<O A$ for all points below the bisectrix. Therefore for any point $B$ of the abscissa to the right of point $e=2.71 \ldots, B C<B D$, which proves the Lemma for points $B$ on the interval $(e, \infty)$. On the segment $[1, e]$ it is true because $\mu(x)$ increases, while $y(x)$ decreases, and $\mu(e)=e^{1 / e}<e$.

It is worth noting that although $x^{y}=y^{x}$ all along the locus, the actual values of $x^{y}$ or $y^{x}$ vary: just take a look at the graph of $w(x)=x^{y(x)}=(y(x))^{x}$ (Figure 4): 


$$
\text { "tmcs-gofen" — 2013/10/21 — 15:20 — page } 249 \text { — \#9 }
$$

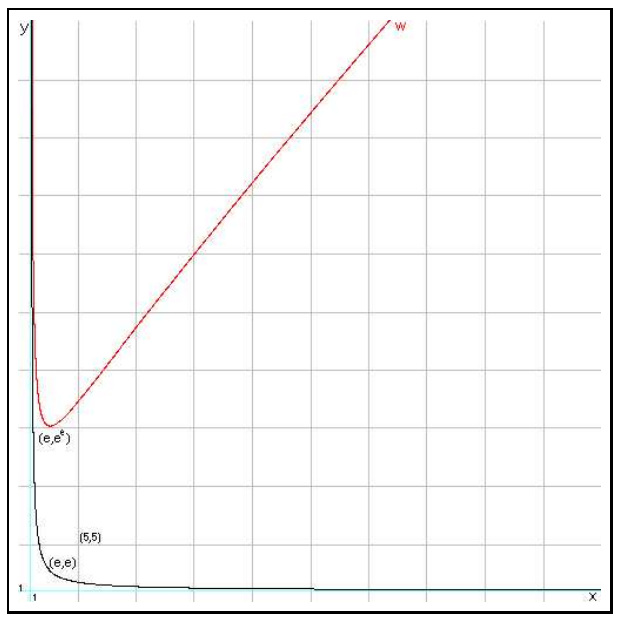

Figure 4. Non-trivial solution $y(x)$ of the equation $x^{y}=y^{x}$ (in black). Function $w(x)=x^{y(x)}=(y(x))^{x} \quad$ (in red)

To determine where is the minimum of $w(x)$, consider

$$
w^{\prime}(x)=x^{y}(\ln x) y^{\prime}+y x^{y-1}=w\left(y^{\prime} \ln x+\frac{y}{x}\right)
$$

and observe that $\left.y^{\prime}\right|_{x=e}=-1$ so that the $w^{\prime}$ changes the sign at this point $\left(\left.w^{\prime}\right|_{x=y=e}=0\right)$. So the minimum value of $x^{y}$ is $e^{e}=15.1542 \ldots$ reached at the point $x=e$.

It is easy to prove that $w(x)$ has a vertical asymptote $x=1$, yet we do not know yet whether $w(x)$ has an inclined asymptote. Numerical experiments with $w(x)$ provide a hint that an inclined asymptote $w(x)=x$ possibly exists. Also the observation over the value $w^{\prime \prime}(x)$ reveals that (unlike in a hyperbola) $w^{\prime \prime}$ changes its sign from positive to negative between 8 and 9 .

The remarkable proximity of the hyperbola $h(x)$ and the curve of commuting powers $y(x)$ is worse of a closer look.

LEMma 2. $y(x) \geq h(x)$ in the neighborhood of the point $x=e$.

Proof. $h(e)=y(e)=e$, and $h^{\prime}(e)=y^{\prime}(e)=-1$, however $h^{\prime \prime}(e)=\frac{2}{e-1}=$ $1.1639 \ldots$ while $y^{\prime \prime}(e)=2 \frac{5}{3 e}=\frac{10}{3 e}=1.2262 \ldots$ Therefore $h(x)<y(x)$ in some neighborhood of the point $x=e$. 
Conjecture 1. $y(x)>h(x)$ in the intervals $(1, e)$ and $(e, \infty)$.

We do not know the proof. As it follows from the parametric formula (2.2), with the notation $u=1+\frac{1}{t}, x=u^{t}$ and $y=u^{t+1}$. In order to prove the Conjecture, it is sufficient to prove that the function $\left(u^{t+1}-1\right)\left(u^{t}-1\right)$ never reaches the value $(e-1)^{2}$, or that it is monotonous.

If the inequality $y(x)>h(x)$ is proven, the following interesting inequalities would follow:

$$
x^{\frac{(e-1)^{2}}{x-1}+1} \leq\left(\frac{(e-1)^{2}}{x-1}+1\right)^{x} \quad \text { or } \quad\left(\frac{(e-1)^{2}}{x-1}+1\right) \ln x \leq x \ln \left(\frac{(e-1)^{2}}{x-1}+1\right) .
$$

\section{Associating powers}

Now consider triplets $(x, y, z)$ of real numbers $x, y, z>0$ for which

$$
x^{\left(y^{z}\right)}=\left(x^{y}\right)^{z}
$$

or after applying logarithm to both sides of the equation

$$
y^{z} \ln x=z \ln x^{y}=z y \ln x .
$$

The case when $\ln x=0$ delivers a trivial solution - a set of triplets $(1, y, z)$ for any $y, z>0$. The non-trivial solutions therefore corresponds to $\ln x \neq 0$ so that we can get rid of the factor $\ln x$ obtaining the transcendental equation defining the function $y(z)$ explicitly (unlike the implicit equation (2.1)): $y^{z}=y z$, or $y^{z-1}=z$, and finally

$$
y=z^{\frac{1}{z-1}} \quad(z \neq 1)
$$

The function $y(z)$ is undefined at the point $z=1$ albeit holomorphic at its neighborhood.

Theorem 4. The functions $y(z)$ and $u(z)=\ln y(z)$ can be holomorphically extended over the point $z=1$.

Proof. Observe that the following limit exists

$$
\lim _{z \rightarrow 1} u(z)=\lim _{z \rightarrow 1} \ln y(z)=\lim _{z \rightarrow 1} \frac{\ln z}{z-1}=\lim _{z \rightarrow 1} \frac{1}{z} / 1=1
$$


(applying L'Hôpital rule). Therefore $u(z)$ can be continuously extended over the point $z=1$ and therefore, according to the Riemann's theorem, can be also holomorphically extended into this point. Then $y(z)=e^{u(z)}$ can be holomorphically extended into the point $z=1$, and $y(1)=e$.

Conclusion 1. The locus of points $(x, y, z)$ for which raising to power is associative is such that either pairs $(y, z)$ belong to the curve $y=z^{\frac{1}{z-1}}$ for an arbitrary $x \neq 1$, or for $x=1$ variables $y, z$ are arbitrary.

Now having the complete answer for which set of triplets $(x, y, z)$ powers associate, we want to take a closer look at the function $y=z^{\frac{1}{z-1}}$.

The function $y(z)$ satisfies a few other known equations: the non-differential equation

$$
(z-1) \ln y-\ln z=0
$$

is most convenient for applying AD to evaluate the Taylor coefficients. And $y(z)$ satisfies the differential equations

$$
\begin{aligned}
& 0=y^{\prime} z(z-1)^{2}-y(z-1)+y z \ln z ; \quad y^{\prime}=y \frac{(z-1)-z \ln z}{(z-1)^{2}} \\
& 0=y^{\prime \prime} y z^{2}(z-1)-\left(y^{\prime}\right)^{2} z^{2}(z-1)+2 y^{\prime} y z^{2}+y^{2}
\end{aligned}
$$

also singular at $z=1$. The equation (3.4) yields the value $\left.y^{\prime}\right|_{z=1}=-\left.\frac{1}{2} y\right|_{z=1}=-\frac{e}{2}$ at this point.

It would be inefficient to evaluate the Taylor coefficient by differentiating the complicated polynomial ODE (3.4). Instead, we differentiate the simple implicit equation (3.3) applying again the formulas of $\mathrm{AD}(2.5)$, as it was done in the previous section. In so doing we obtain the following recursive formula

$$
y^{[n]}=\frac{1}{n} \sum_{i=0}^{n-1} y^{[i]} \frac{(-1)^{n-i}(n-i)}{(n-i+1)}, \quad n>0 .
$$

In particular: $\left.y^{[1]}\right|_{z=1}=-\frac{e}{2},\left.y^{[2]}\right|_{z=1}=\frac{11 e}{24},\left.y^{[3]}\right|_{z=1}=-\frac{89}{72} e$.

For this recursive formula (3.5) we also do not know the convoluted solution, nor can we estimate the convergence radius judging by $y^{[n]}$. We do know however that the convergence radius is nonzero because we have proved that $y(z)$ is holomorphic in the previous Theorem.

Below is the graph of $y(z)$ : 


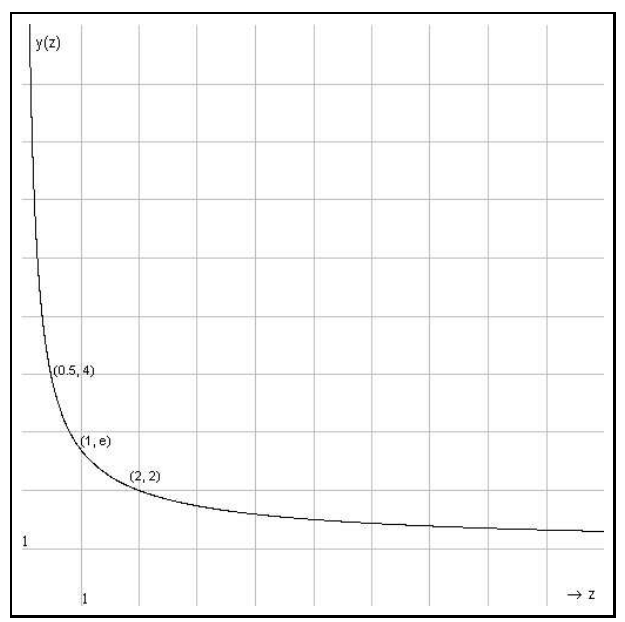

Figure 5. The function $y(z)$ of the associating powers

Here are several points satisfying the equation $y^{z}=y z$ or $y(z)=z^{\frac{1}{z-1}}$ : $(0.5,4),(1, e),(2,2)$.

\section{The conclusion about the special points in the two functions}

In [3] we studied a new type of special points in holomorphic functions such as the point $t=0$ in $x(t)=\frac{\sin t}{t}$. The specialty of such points is in that the function $x(t)$ cannot satisfy any regular at $t=0$ rational $n$-order ODE $x^{(n)}=R\left(t, x, \ldots, x^{(n-1)}\right)$, nor can it satisfy any polynomial ODE $x^{(n)}=$ $P\left(t, x, \ldots, x^{(n-1)}\right)$. The Taylor coefficients of those functions at the special points are sequences of particular rational numbers - fractions in smallest terms whose denominators infinitely grow and take the values of prime numbers. The method of proof relied on the fact that for big enough primes in the denominator, the fraction cannot be cancelled by the other fractions in the sum.

The Taylor expansions at the special points for both function considered in this paper do not fit the format required in [3] at least because of presence of $e$ in all terms. It is possible to get rid of $e$ with an appropriate replacement of the variables, so that only rational expressions remain in both expansions. Yet even then we do not know how to identify fractions in smallest terms with growing prime denominators in those expansions. 
Therefore, the issue whether the singular ODEs (2.3) and (3.4) may be replaced with regular rational ODEs remains open.

\section{Acknowledgement}

I take an opportunity to express my deep appreciation to Lajos Lòczi who kindly provided me with his survey on the functions of commuting and associating powers, and particularly for his help in computing the multi-digit fractions evaluating the terms in the Taylor expansion of the commuting powers at the special point $(e, e)$.

\section{Appendix 1}

The graphs and ODEs referred in this article can be replayed in a free demoversion of an advance ODE solver [6] implementing the modern Taylor method for integration of ODEs. One of many features of this software is that it can start integration even at a singular point of the ODEs if the solution is regular at this point and the special Taylor expansion of the solution is provided (from other sources). Thanks to this feature, the software can deal with the special ODEs studied here and pre-loaded into the distribution package [6].

After installing the demo-version according to the instructions, go to File/Open Script menu item, navigate to Samples/SpecPoint folder, and then the following ODEs are available for playing and further experiments.

(1) Commuting powers in the file $x^{\wedge} y=y^{\wedge} x 1 . s c r$. It graphs the ODE (2.3);

(2) Commuting powers in the file $x^{\wedge} y=y^{\wedge} x$ 2.scr. It graphs the cumbersome ODE $(2.4)$;

(3) Commuting powers in the file $x^{\wedge} y=y^{\wedge} x$ Param.scr. It graphs the parametric curve (2.2);

(4) Associating powers in the file $z^{\wedge}\left(y^{\wedge} x\right)=\left(z^{\wedge} y\right)^{\wedge} x . s c r$. It graphs the equation (3.2). The script graphs the curve on the segment $[1, \infty)$. In order to obtain the part on the interval $(0,1]$ press Restart button, set the switch Backward and integrate pressing button More.

(5) Solution of the ODE $y^{\prime \prime}=y / x$ in the script file $x 2=x$ Divt.scr;

(6) The Bessel functions in the script files Bessel-0-2.scr, Bessel-1-2.scr, Bessel0 -3.scr;

(7) Function $\frac{\sin t}{t}$ in the script file $\sin (t)$ Divt.scr. 


\section{References}

[1] L. Lòczi, Two centuries of the equations of commutativity and associativity of exponentiation, Teaching Math. and Comp. Sci. 1, no. 2 (2003), 219-233.

[2] A. Gofen, The ordinary differential equations and automatic differentiation unified, Complex Variables and Elliptic Equations 54, no. 9 (September 2009), 825-854, (Also here: http://www.ski.org/gofen/UnifiedView.pdf).

[3] A. Gofen, Unremovable 'Removable' Singularities, Complex Variables and Elliptic Equations 53, no. 7 (2008), 633-642, (Also here: http://www.ski.org/gofen/UnremovSingularity.pdf).

[4] G. Corliss ed., Automatic Differentiation: Applications, Theory, and Tools, Lecture Notes in Computational Science and Engineering, Springer, 2005.

[5] R. E. Moore, Interval Analysis, Prenitce-Hall, Englewood Cliffs, N.Y., 1966.

[6] A. Gofen, http://www.ski.org/gofen/TaylorMethod.htm.

ALEXANDER GOFEN

SAN FRANCISCO

USA

E-mail: galex@ski.org

(Received March, 2013) 


\title{
Correction to Gofen (2013)
}

\author{
Alexander Gofen
}

In the article "Powers which commute or associate as solutions of ODEs?" by Alexander Gofen (Teaching Mathematics and Computer Science, 2013, 11(2), 241-254. https://doi.org/10.5485/TMCS.2013.0347), there was an error in Conjecture 1 (p. 250), and consequently, in the References (p. 254).

The first sentence of Conjecture 1, "We do not know the proof." should be completed with the following footnote:

In 2020, Lajos Lóczi proved this conjecture (Lóczi, 2021).

Additionally, one more item should be included in the References:

[7] L. Lóczi, Explicit and recursive estimates of the Lambert $\mathrm{W}$ function, https://arxiv.org/abs/2008.06122, (2021).

The URLs in items [2], [3], and [6] of the References should be updated as follows:

[2] http://TaylorCenter.org/gofen/UnifiedView.pdf

[3] http://TaylorCenter.org/gofen/UnremovSingularity.pdf

[6] http://TaylorCenter.org/gofen/TaylorMethod.htm 